International Journal of Pure and Applied Mathematics

Volume 86 No. 3 2013, 521-525

ISSN: 1311-8080 (printed version); ISSN: 1314-3395 (on-line version)

url: http://www.ijpam.eu

doi: http://dx.doi.org/10.12732/ijpam.v86i3.6

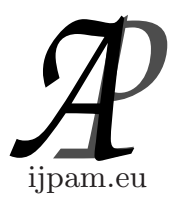

\title{
ON THE SLOPE OF \\ THE SCHUR FUNCTOR OF A VECTOR BUNDLE
}

\author{
Elena Rubei
}

Dipartimento di Matematica e Informatica "U. Dini"

Viale Morgagni 67/A, 50134, Firenze, ITALY

\begin{abstract}
We prove that, for any complex vector bundle $E$ of rank $e$ on a compact Kähler manifold $X$, we have that $\mu\left(S^{\lambda} E\right)=|\lambda| \mu(E)$ for any $\lambda=$ $\left(\lambda_{1}, \ldots, \lambda_{e-1}\right)$ with $\lambda_{i} \in \mathbb{N}$ and $\lambda_{1} \geq \ldots \geq \lambda_{e-1}$, where $|\lambda|=\lambda_{1}+\ldots+\lambda_{e-1}$, the symbol $S^{\lambda}$ denotes the Schur functor and $\mu$ is the slope. This result has already been stated, without proof, by Ottaviani in 1995.
\end{abstract}

AMS Subject Classification: 19L10, 55R10

Key Words: slope, Schur functors

\section{Introduction}

In this short note we prove that for any complex vector bundle $E$ of rank $e$ on a compact Kähler manifold $X$, we have that

$$
\mu\left(S^{\lambda} E\right)=|\lambda| \mu(E)
$$

for any $\lambda=\left(\lambda_{1}, \ldots, \lambda_{e-1}\right)$ with $\lambda_{i} \in \mathbb{N}$ and $\lambda_{1} \geq \ldots \geq \lambda_{e-1}$, where $|\lambda|=$ $\lambda_{1}+\ldots+\lambda_{e-1}$, the symbol $S^{\lambda}$ denotes the Schur functor and $\mu$ is the slope. This result has already been stated, without proof, in [3] (and used in the recent paper [4]) In [1] it is stated and proved only in the case of the exterior powers.

Received: March 20, 2013

(c) 2013 Academic Publications, Ltd. url: www.acadpubl.eu 
Since the lack of a proof of the general case in the literature, we expose here a complete proof.

Among the literature on the subject, we quote also [5]: in it, the author calculated the Chern characters of the symmetric powers and of the exterior powers of a vector bundle. It seems difficult to generalize his results to any Schur functors of a vector bundle.

\section{Notation and Recalls}

Let $\lambda=\left(\lambda_{1}, \ldots, \lambda_{e-1}\right)$ with $\lambda_{i} \in \mathbb{N}$ and $\lambda_{1} \geq \ldots \geq \lambda_{e-1}$. For any $V$ complex vector space of dimension $n$, the symbol $S^{\lambda} V$ will denote the Schur representation $(S L(V)$-representation) associated to $\lambda$ (see Lecture 6 in $[2])$.

The $S^{\lambda} V$ are irreducible $S L(V)$-representations and it is well-known that all the irreducible $S L(V)$-representations are of this form.

We recall that Pieri's formula says that, if $\nu=\left(\nu_{1}, \nu_{2}, \ldots\right)$ is a partition of a natural number $d$ with $\nu_{1} \geq \nu_{2} \geq$.. and $t$ is a natural number, then

$$
S^{\nu} V \otimes S^{t} V=\oplus_{\gamma \in \Gamma} S^{\gamma} V
$$

as $S L(V)$-representation, where $\Gamma$ is the set of all the partitions $\gamma=\left(\gamma_{1}, \gamma_{2}, \ldots\right)$ with $\gamma_{1} \geq \gamma_{2} \geq \ldots$ of $d+t$ whose Young diagrams are obtained from the Young diagram of $\nu$ adding $t$ boxes not two in the same column.

Notation 1. If $E$ is a complex vector bundle on a compact Kähler manifold $X$, then $\mu(E)$ will denote the slope of $E$, i.e. the degree of $E$ divided by the rank of $E$.

\section{The Proof}

Theorem 2. For any complex vector bundle $E$ of rank $e$ on a compact Kähler manifold $X$, we have that

$$
\mu\left(S^{\lambda} E\right)=|\lambda| \mu(E)
$$

for any $\lambda=\left(\lambda_{1}, \ldots, \lambda_{e-1}\right)$ with $\lambda_{1} \geq \ldots \geq \lambda_{e-1} \geq 0$, where $|\lambda|=\lambda_{1}+\ldots+\lambda_{e-1}$.

Proof. - First we prove the result in the case $\lambda=(m, 0, \ldots, 0)$, i.e. in the case of the symmetric powers of $E$. Obviously

$$
\mu\left(S^{m} E\right)=\frac{\operatorname{deg}\left(S^{m} E\right)}{r k\left(S^{m} E\right)}=\frac{\operatorname{deg}\left(S^{m} E\right)}{\left(\begin{array}{c}
e+m-1 \\
m
\end{array}\right)},
$$


so we have to prove that

$$
\operatorname{deg}\left(S^{m} E\right)=\left(\begin{array}{c}
e+m-1 \\
e
\end{array}\right) \operatorname{deg}(E) .
$$

We prove it by induction on $e$. The case $e=1$ is trivial. By the splitting principle, we can suppose that

$$
E=E_{1} \oplus \ldots . \oplus E_{e}
$$

where $r k\left(E_{i}\right)=1$ for $i=1, \ldots, e$. So

$$
\begin{gathered}
\operatorname{deg}\left(S^{m} E\right)=\operatorname{deg}\left(\oplus_{i_{1}, \ldots, i_{e} \in \mathbb{N}, i_{1}+\ldots .+i_{e}=m} S^{i_{1}} E_{1} \otimes \ldots \otimes S^{i_{e}} E_{e}\right) \\
=\sum_{i_{1}, \ldots, i_{e} \in \mathbb{N}, i_{1}+\ldots .+i_{e}=m} \operatorname{deg}\left(S^{i_{1}} E_{1} \otimes \ldots \otimes S^{i_{e}} E_{e}\right) \\
=\sum_{i_{1}, \ldots, i_{e} \in \mathbb{N}, i_{1}+\ldots .+i_{e}=m}\left[\operatorname{deg}\left(S^{i_{1}} E_{1}\right)+\ldots+\operatorname{deg}\left(S^{i_{e}} E_{e}\right)\right] \\
=\sum_{i_{1}, \ldots, i_{e} \in \mathbb{N}, i_{1}+\ldots .+i_{e}=m}\left[i_{1} \operatorname{deg}\left(E_{1}\right)+\ldots .+i_{e} \operatorname{deg}\left(E_{e}\right)\right] .
\end{gathered}
$$

Observe that coefficients of $\operatorname{deg}\left(E_{1}\right), \ldots ., \operatorname{deg}\left(E_{e}\right)$ in the above formula must be equal. Besides the sum of the coefficients of $\operatorname{deg}\left(E_{1}\right), \ldots, \operatorname{deg}\left(E_{e}\right) \operatorname{must}$ be

$$
\sum_{i_{1}, \ldots, i_{e} \in \mathbb{N}, i_{1}+\ldots+i_{e}=m} i_{1}+\ldots+i_{e},
$$

that is $\left(\begin{array}{c}e-1+m \\ m\end{array}\right) m$. Therefore the coefficient of $\operatorname{deg}\left(E_{i}\right)$ for any $i=1, \ldots, e$ must be $\frac{m}{e}\left(\begin{array}{c}e-1+m \\ m\end{array}\right)$. Thus we get

$$
\begin{aligned}
\operatorname{deg}(E)=\frac{m}{e}\left(\begin{array}{c}
e-1+m \\
m
\end{array}\right)\left(\operatorname{deg}\left(E_{1}\right)+\ldots .+\operatorname{deg}\left(E_{e}\right)\right) & \\
& =\frac{m}{e}\left(\begin{array}{c}
e-1+m \\
m
\end{array}\right) \operatorname{deg}(E),
\end{aligned}
$$

as we wanted to prove.

- Now we prove the result in general by induction on the number of the rows of the Young diagram of $\lambda$. If the number of the rows is 1, we already know the result.

So suppose that the statement holds for $S^{\alpha} E$ with the number of the rows of $\alpha$ less or equal than $k$. 
We want to prove the statement when the number of the rows is less or equal than $k+1$; we show it by induction on the number $t$ of the elements of the $(k+1)$-th row. If $t=0$ we know the statement by induction assumption. So suppose that the Young diagram of $\lambda$ has $k+1$ rows and $t$ elements in the $(k+1)$-th row.

We define $\nu$ to be the Young diagram we get from $\lambda$ by deleting the last row.

Consider Pieri's formula applied to $S^{\nu} E \otimes S^{t} E$ :

$$
S^{\nu} E \otimes S^{t} E=\oplus_{\gamma \in \Gamma} S^{\gamma} E
$$

where $\Gamma$ is the set of all the partitions $\gamma=\left(\gamma_{1}, \gamma_{2}, \ldots\right)$ with $\gamma_{1} \geq \gamma_{2} \geq \ldots$ of $|\nu|+t$ whose Young diagrams are obtained from the Young diagram of $\nu$ adding $t$ boxes not two in the same column. Obviuosly $\lambda \in \Gamma$, so we can write $\Gamma=\{\lambda\} \cup \Gamma^{\prime}$. We have:

$$
\mu\left(S^{\nu} E \otimes S^{t} E\right)=\mu\left(\oplus_{\gamma \in \Gamma} S^{\gamma} E\right),
$$

therefore

$$
\mu\left(S^{\nu} E\right)+\mu\left(S^{t} E\right)=\mu\left(\oplus_{\gamma \in \Gamma^{\prime}} S^{\gamma} E \oplus S^{\lambda} E\right),
$$

then, by induction assumption,

$$
|\nu| \mu(E)+t \mu(E)=\mu\left(\oplus_{\gamma \in \Gamma^{\prime}} S^{\gamma} E \oplus S^{\lambda} E\right) .
$$

Hence we get:

$$
(|\nu|+t) \mu(E)=\frac{\sum_{\gamma \in \Gamma^{\prime}} \operatorname{deg}\left(S^{\gamma} E\right)+\operatorname{deg}\left(S^{\lambda} E\right)}{r k\left(S^{\nu} E \otimes S^{t} E\right)}
$$

Therefore

$$
r k\left(S^{\nu} E \otimes S^{t} E\right)(|\nu|+t) \mu(E)=\sum_{\gamma \in \Gamma^{\prime}} \operatorname{deg}\left(S^{\gamma} E\right)+\operatorname{deg}\left(S^{\lambda} E\right) .
$$

Hence

$$
\begin{aligned}
\operatorname{deg}\left(S^{\lambda} E\right) & =r k\left(S^{\nu} E \otimes S^{t} E\right)(|\nu|+t) \mu(E)-\sum_{\gamma \in \Gamma^{\prime}} \operatorname{deg}\left(S^{\gamma} E\right) \\
& =r k\left(S^{\nu} E \otimes S^{t} E\right)(|\nu|+t) \mu(E)-\sum_{\gamma \in \Gamma^{\prime}} r k\left(S^{\gamma} E\right) \mu\left(S^{\gamma} E\right) \\
& =r k\left(S^{\nu} E \otimes S^{t} E\right)(|\nu|+t) \mu(E)-\sum_{\gamma \in \Gamma^{\prime}} r k\left(S^{\gamma} E\right)|\gamma| \mu(E)
\end{aligned}
$$




$$
\begin{array}{r}
=\mu(E)(|\nu|+t)\left[r k\left(S^{\nu} E \otimes S^{t} E\right)-\sum_{\gamma \in \Gamma^{\prime}} r k\left(S^{\gamma} E\right) \mid=\mu(E)(|\nu|+t) r k\left(S^{\lambda} E\right)\right. \\
=r k\left(S^{\lambda} E\right)|\lambda| \mu(E)
\end{array}
$$

where the last but three equality holds by induction assumption (induction on $t$ ) and the last equality and the last but two equality hold because $|\lambda|=|\gamma|=$ $|\nu|+t$.

\section{References}

[1] V. Ancona, G. Ottaviani, Stability of special instanton bundles on $\mathbf{P}^{2 n+1}$, Trans. Amer. Math. Soc., 341, No. 2 (1994), 677-693, doi: 10.1090/S00029947-1994-1136544-9.

[2] W. Fulton, J. Harris, Representation Theory, A First Course Graduate Texts in Mathematics, USA, Springer Verlag (1991), doi: 10.1007/978-14612-0979-9.

[3] G. Ottaviani Varietà proiettive di codimensione piccola Quaderni INDAM, Aracne, Italy (1995), ISBN 9788879991193.

[4] E. Rubei, Stability of homogeneous bundles on $\mathbf{P}^{3}$, Geometriae Dedicata, 158, No. 1 (2012), 1-21, doi: 10.1007/s10711-011-9617-9.

[5] D. Svrtan, New plethysm operation, Chern characters of exterior and symmetric powers with applications to Stiefel-Whitney classes of grassmannians, Theoretical Computer Science, 117 (1993), 289-301, doi: 10.1016/0304-3975(93)90320-S. 
